\title{
Numerical Analysis of Blade Geometry Generation Techniques for Centrifugal Compressors
}

\author{
Florin lancu, John Trevino, and Steven Sommer \\ Johnson Controls Inc., P.O. Box 1592-191A, York, PA 17405-1592, USA
}

Received 22 August 2007; Accepted 22 November 2007

Recommended by Seung Jin Song

It is a known fact that machined impellers result in improved compressor performance compared to cast impellers of the same design. The performance improvements can be attributed to better surface finish, more accurate geometric definition (tighter dimensional tolerances), well-defined edges, and the lack of blade tip fillet on shrouded impellers. In addition, it has been observed through experimental investigations that the construction method of the impellers has an impact on performance. This paper presents computational fluid dynamic investigations of two types of impellers, with blade surfaces generated using straightline elements (SLEs) and CAD arbitrary definitions. Because there are many different mathematical definitions that CAD tools employ for curves, the resulting arbitrary blade surface is not unique. The numerical results will help understand the causes of the performance differences as well as the effects of SLE blades on the flow through the impeller. Input conditions for computational dynamic simulations are based on experimental results. All references to experimental data in the present paper are for cast impellers. Therefore, the differences in performance are attributed to blade definition (SLE versus other) and not to differences resulting from manufacturing methods.

Copyright (C) 2007 Florin Iancu et al. This is an open access article distributed under the Creative Commons Attribution License, which permits unrestricted use, distribution, and reproduction in any medium, provided the original work is properly cited.

\section{INTRODUCTION}

Although there is a large number of publications describing the numerical simulation of centrifugal compressor impellers and other publications dealing with manufacturing of machined impellers, there is not too much information describing the impact of the SLE surfaces on the flow field inside the centrifugal compressor impeller and its performance. For flank-milled machined impellers, a hub and shroud blade profile is connected by predetermined SLE, which would correspond to a tool path, to generate the blade surface according to the design intent of the compressor engineer. For cast impellers, the method of connecting hub and shroud blade profile points leads to an arbitrary surface definition and is dependent upon a designer's interpretation of blade profile data and/or the solid model, as well as the CAD software. Although the shapes of the hub and shroud profiles are preserved, the resulting blade surface defined by connecting these two profiles may not correspond to the design intent of the compressor engineer. Because the blade surface deviates from the design intent, the compressor performance can deteriorate. Foundries rely on a full 3D design model to create tooling for cast impellers, as opposed to hub and shroud profiles typically required of a 5 -axis machining program. Therefore, these construction differences become significant for cast impellers.

Two impellers were analyzed using a commercially available computational fluid dynamic (CFD) code, and the results were first validated against experimental data. All experimental data presented in this paper are for impellers manufactured utilizing an investment cast process from the same supplier. After establishing confidence in the numerical models, a comparative study between the SLE and the CAD generated impeller (denominated in this paper as "curved blade impeller" as opposed to the SLE impeller) was performed. An attempt to pinpoint the main differences in geometries that lead to enhance the performance of the SLE blade is the main topic of the paper and these results are presented next.

CFD analysis of centrifugal impellers is not a new technique; in fact it is common practice to perform such analysis as part of the design process. Several examples of centrifugal compressor component studies are found in the extant literature. Pitkänen and his research group delivered several conclusions after their CFD analysis of a centrifugal compressor [1]: the most complete method for simulation of turbomachinery is to rotate the mesh describing the rotor and 


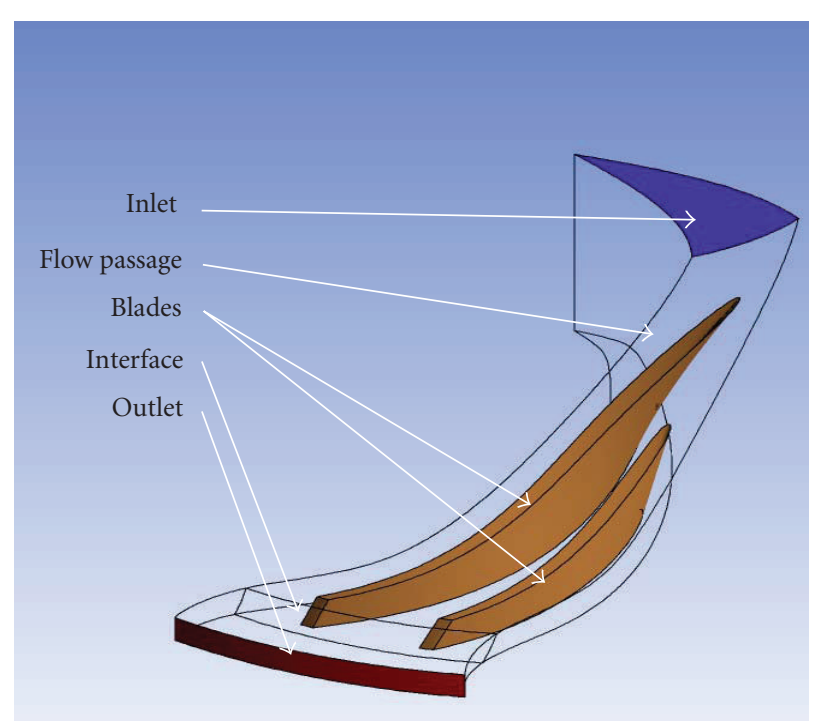

FIGURE 1: Schematic of a single passage of a centrifugal impeller.

connect it to the stationary part through a sliding mesh interface; quasi-steady-state simulation is almost as accurate as the time-dependent calculation. Flathers and Bache [2] studied the radial forces and flow field inside a centrifugal compressor. Using the $3 \mathrm{D}$ CFD viscous code TASCFlow, they were able to simulate the gasdynamic process of the whole centrifugal compressor stage (impeller, diffuser, and volute) and obtain results that accurately matched the experimental ones. The same commercial code was used by Koumoutsos and his team in the study of unsteady flows in centrifugal compressors [3]. Their conclusions suggest that even though the unsteady simulation is much more accurate and captures better the phenomena inside the centrifugal compressor stage, a steady solution will provide results accurate enough to be used as valid predictions in the design process. Highly successful were Shah and Bartos [4] in predicting performance of centrifugal compressors using the same CFD code TASCFlow. Using a structured grid and simplified blade geometry, the code was able to predict pressure and temperature distribution within 1\% from the experimental values.

\section{NUMERICAL SIMULATIONS}

The numerical model was created and analyzed using the ANSYS CFX-10 package (formerly known as TASCFlow). From the solid model of the impeller, a negative mold was created that describes the flow passages. This solid model was the base geometry for creating the finite volume mesh. Due to periodicity features of the impeller geometry, only one passage containing one main blade and one splitter blade was modeled. To accommodate the full development of the flow, a portion of the radial vaneless diffuser was also modeled as a stationary mesh. Some details of the model geometry can be seen in Figure 1.

The model uses an unstructured mesh made of tetrahedrons and triangular prisms, the final size of the mesh being decided upon the results of a grid sensitivity study, with a number of elements ranging from approximately 0.5 million to 3.5 million. The mesh comprises several thin layers of elements along the boundaries to accommodate the phenomena occurring inside the flow boundary layers. The model has a rotating mesh, the impeller part, and a stationary one, the diffuser region. The interface between these two regions was simulated as either frozen rotor type or stage type. The stage interface delivers slightly more realistic results since it uses a rotating mesh into a fixed reference frame, while the frozen rotor uses a rotating reference frame and stationary mesh.

The inlet boundary conditions were total pressure and total temperature, while for the outlet, mass flow rate was chosen. The inlet turbulence model was based on intensity and length scale with a 5\% fractional intensity, and Eddy length scale equals approximately $10 \%$ of the impeller eye diameter. The walls were modeled as adiabatic and hydrodynamic smooth, while the heat transfer from fluid zone to fluid zone being considered based on total energy.

The fluid used for analysis was the available R-134a in the Redlich-Kwong refrigerant definition supplied by ANSYS CFX. The steady-state solver uses a high-resolution advection scheme, convergence being attained when the mass and momentum root-mean-square (RMS) residuals drop below $10^{-5}$. Two turbulence models have been investigated: the $k-\varepsilon$ and the shear stress transport, the first one being chosen for introducing a more realistic degree of viscosity.

\section{EXPERIMENTAL INVESTIGATIONS}

The test was set up according to the guidelines of the power test code (PTC) no. 10 for centrifugal and axial flow compressors and blowers [5]. For compressor testing, a closed system was used with refrigerant R134a as the compression fluid.

The refrigerant system is a condensing-type run-around loop, most of the discharge gas being expanded to a lower pressure in a suction desuperheater and cooled by injecting liquid refrigerant to obtain the desired compressor inlet condition. The rest of the discharge gas is condensed in a watercooled condenser and the resultant liquid is used for injection.

A cooling water system and a cooling tower are used to maintain a constant refrigerant condensing pressure. The cooling tower removes the heat input from the water circulating pump and the drive motor input to the compressor. The compressor is driven by a constant torque $1500 \mathrm{HP}$ D.C. motor with variable speed control through an electronic torquemeter and speed increaser.

\section{RESULTS AND DISCUSSION}

\subsection{Experimental versus numerical investigations}

Table 1 presents a comparison between efficiency and head increases from the curved blade impeller to the SLE one in the experimental and numerical investigations. Although the results from these two types of investigations cannot be compared directly, a similarity in the trend of the results can be observed. 
TABLE 1: Experimental and numerical comparative results.

\begin{tabular}{|c|c|c|c|c|}
\hline \multirow[t]{2}{*}{ Flow factor } & \multicolumn{2}{|c|}{$\begin{array}{c}\text { Efficiency difference } \\
\text { between SLE and curved }\end{array}$} & \multicolumn{2}{|c|}{$\begin{array}{c}\text { Head difference } \\
\text { between SLE and curved }\end{array}$} \\
\hline & EXP & NUM & EXP & NUM \\
\hline 0.0508 & $0.0 \%$ & $0.0 \%$ & $0.4 \%$ & $0.2 \%$ \\
\hline 0.0630 & $1.0 \%$ & $1.1 \%$ & $5.0 \%$ & $1.4 \%$ \\
\hline 0.0713 & $3.1 \%$ & $1.8 \%$ & $8.3 \%$ & $2.0 \%$ \\
\hline 0.0764 & $6.0 \%$ & $1.9 \%$ & $12.0 \%$ & $2.3 \%$ \\
\hline 0.0788 & $11.4 \%$ & $3.4 \%$ & $17.6 \%$ & $3.4 \%$ \\
\hline
\end{tabular}

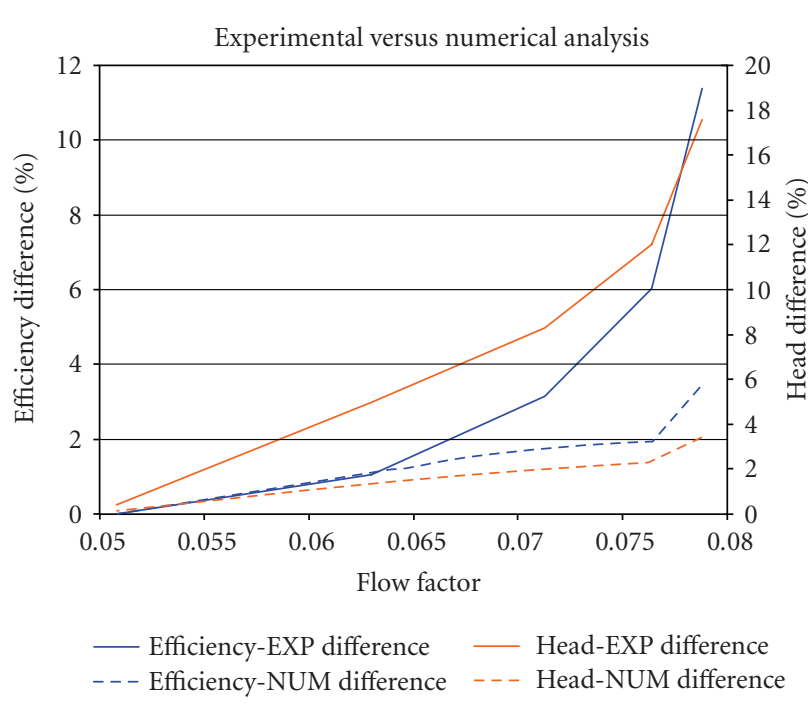

FIgURE 2: Compression efficiency comparative results.

The efficiency and head calculations are similar with the test data and the numerical data, but the domain is different. The compressor was instrumented for flange-to-flange performance, thus the experimental efficiency is based on compressor suction and discharge measurements. In the numerical case, for time considerations, only the impeller and a small fraction of the vaneless diffuser were modeled, thus the compression efficiency is based on impeller inlet and outlet characteristics. Also, the numerical model does not account for various other losses in the system, like heat transfer, leakage, and frictional and other mechanical losses.

Nevertheless, the two sets of data show the same trend: the SLE blade impeller yields a more efficient compression of the refrigerant. Figure 2 shows these two trends of efficiency and head increases. Although the CFD generates much smaller differences between SLE and curved blades than the experimental results, the curves move in the same direction and with the same relative magnitude of each segment.

\subsection{SLE blades versus curved blades}

When examining the numerical results (Table 2), it can be seen that the efficiency differences are generated by small differences in pressure and temperature increases along the

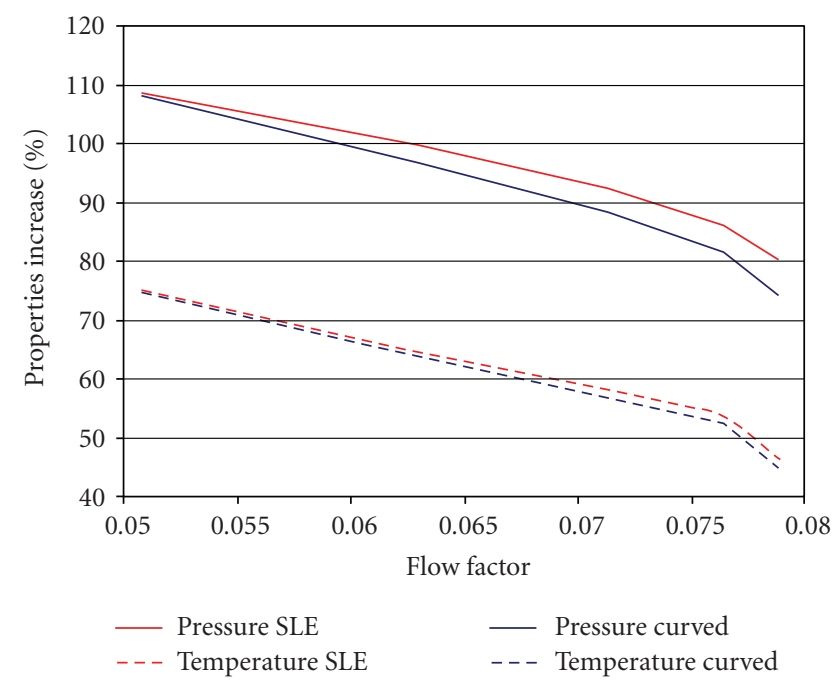

FIgure 3: Pressure and temperature increases for SLE and curved bladed impellers.

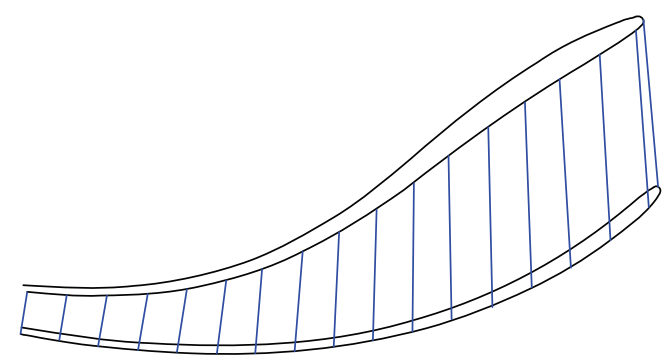

(a)

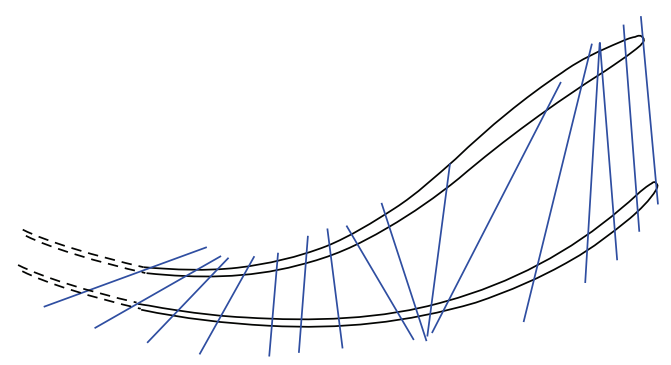

(b)

FIGURE 4: Examples of blade definition by means of straight-line elements. 
TABLE 2: Comparative properties of SLE and curved blades analyses.

\begin{tabular}{lcccc}
\hline Flow factor & \multicolumn{2}{c}{$\begin{array}{c}\text { Pressure increase } \\
\text { (total to static) }\end{array}$} & \multicolumn{2}{c}{$\begin{array}{c}\text { Temperature increase } \\
\text { (total to static) }\end{array}$} \\
\hline 0.0508 & SLE blade & Curved blade & SLE blade & $75.0 \%$ \\
0.0630 & $108.6 \%$ & $108.0 \%$ & $64.5 \%$ & $74.7 \%$ \\
0.0713 & $99.7 \%$ & $96.8 \%$ & $58.3 \%$ & $53.5 \%$ \\
0.0764 & $92.5 \%$ & $88.4 \%$ & $54.0 \%$ & $52.5 \%$ \\
0.0788 & $86.0 \%$ & $81.6 \%$ & $46.6 \%$ & $44.7 \%$ \\
\hline
\end{tabular}

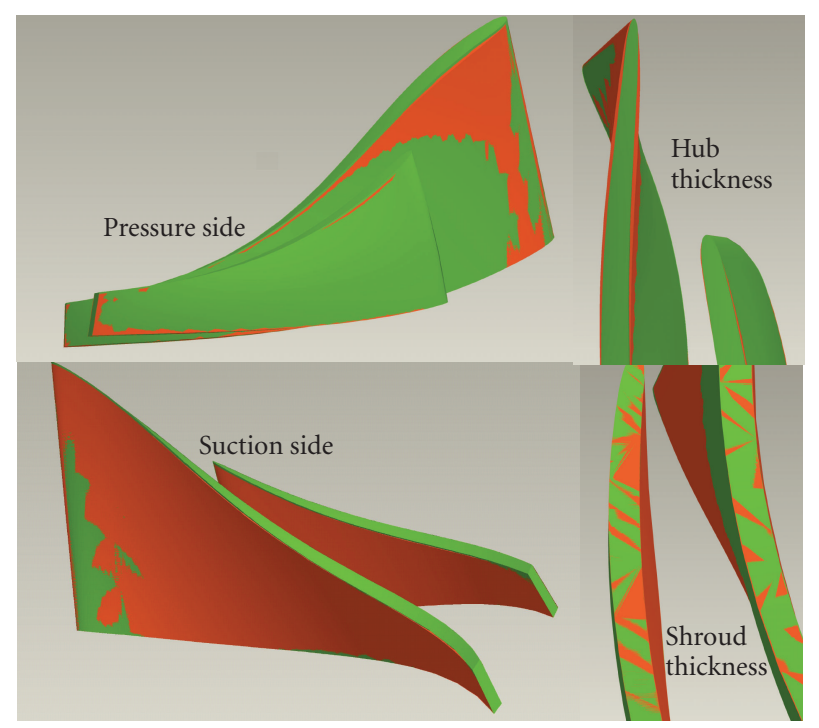

SLE blade

Curved blade

FIGURE 5: Visual comparison of the two impellers' geometries.

flow passage. Both the temperature and pressure increases are higher in the SLE case, but the combination of these two results in higher efficiency. The graph presented in Figure 3 shows that the difference between pressure increases in the SLE and curve cases is higher than the temperature difference, leading to the efficiency difference. Also, the increasing difference of pressure and temperature with flow factor is translated into increased efficiency (presented in Figure 2).

The next step is analyzing the flow fields of the two types of impellers, trying to identify the differences that cause the improvement in performance of one blade versus the other.

The CFD analysis tried to replicate the best way possible the real test data, thus the geometry used to create the numerical model was based on the files that were sent to the investment-cast foundry. The first CAD model was created inadvertently without considering the compressor engineer's design intent of blade element connection, thus resulting in the curved blades. The second CAD model accounts for the design intent SLE blade definition. When making a visual comparison of these geometries, it can be seen that

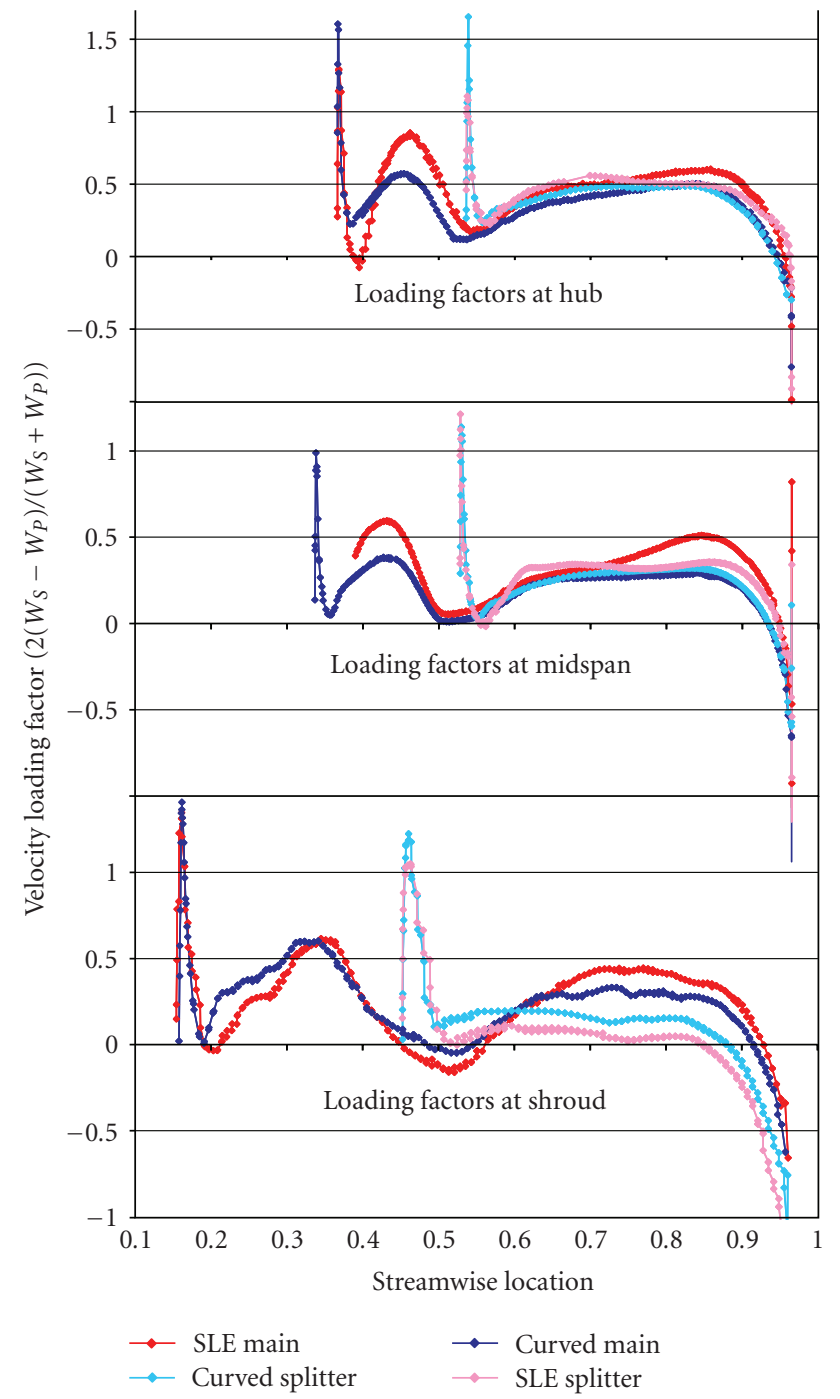

FIGURE 6: Loading factors ( $\mathrm{W}_{S}$ - velocity at suction side, $\mathrm{W}_{P}-$ velocity at pressure side) at flow factor of 0.0713 .

the SLE and the curved blades differ significantly, that is, intriguing for two blades that were supposed to be created following the same set of points that would describe the hub and shroud profile of the blade. Machining software (if 5-axis flank machining were used for manufacturing) would generate a tool path that is close to the original design geometry, 

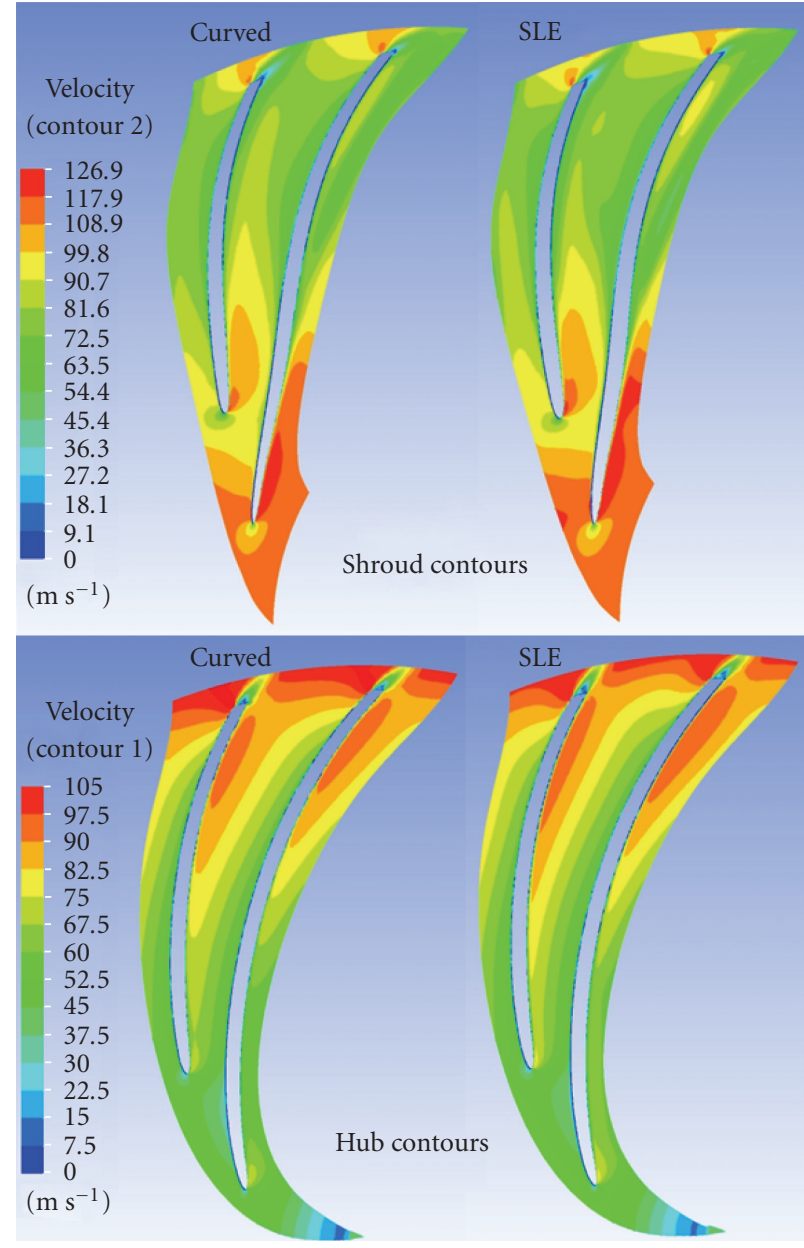

Figure 7: Relative velocity contours along the hub and shroud of the impeller at flow factor of 0.0713 .

but not identical. Here is where that uncertainty of how software will generate curves from points and, in turn, surfaces from curves comes into play.

A study of machined impellers was published by Tsay et al. [6]. As described by Tsay et al., the curves defining the blades, as well as the hub and shroud, are each constructed from a set of points, thus the resultant spline is not unique. This spline that will define the machining tool path is constructed by interpolation through the given points. Although they prove that the resultant impeller geometry is close to design, there is still room for variations.

Figure 4 shows the difference between the two methods. Most CAD software packages will generate lines to connect hub and shroud points located at the same streamwise position (Figure $4(\mathrm{a})$ ) and then interpolate a pressure and a suction surface between these lines. The machining software used on a 5-axis flank milling machine will extend the hub and shroud curves downstream to be able to map the surface defined at Figure 4(a) using only straight lines. These lines are not necessarily intersecting the hub and shroud at the same streamwise location, an example being shown in
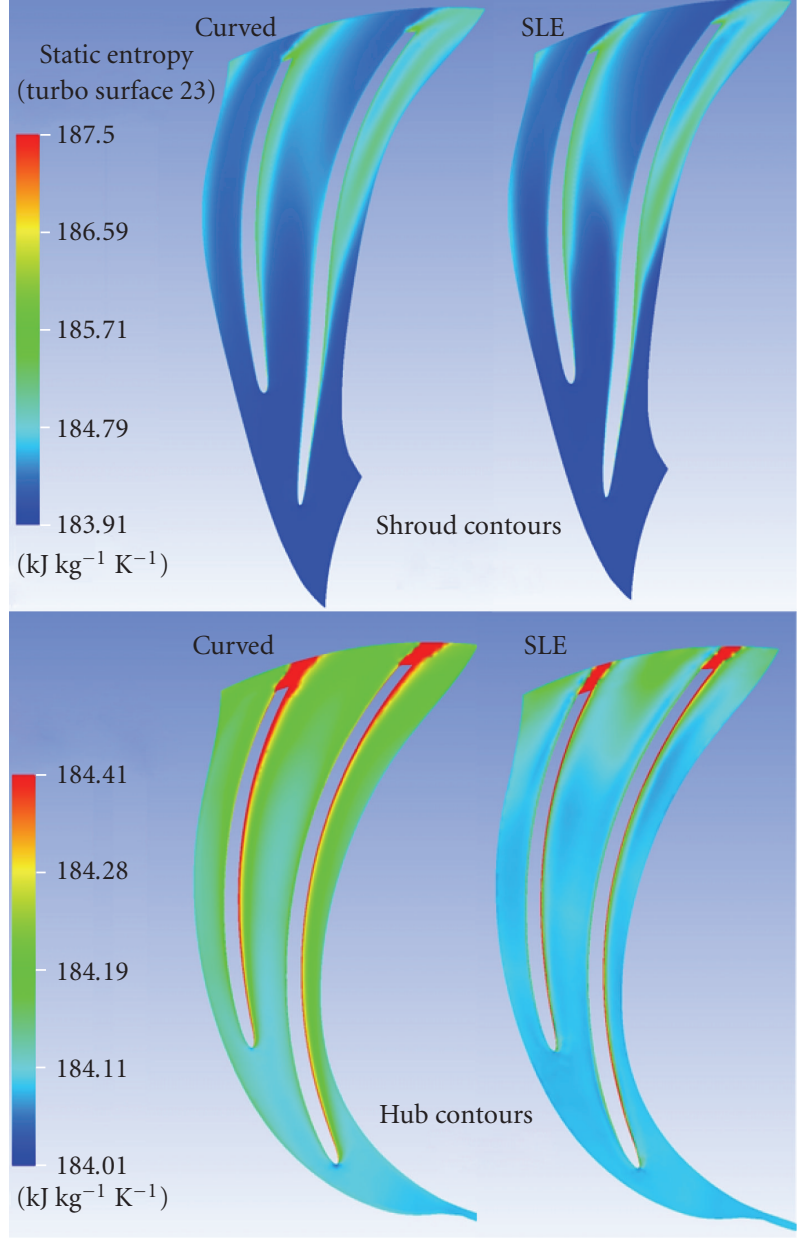

FIGURE 8: Static entropy contours along constant spanwise position at $5 \%$ and $95 \%$ of spanwise distance at flow factor of 0.0713 (scale was upper bounded at $184.41 \mathrm{KJ} / \mathrm{kg} \cdot \mathrm{K}$ ).

Figure 4(b). The part resulting from the machining process will be similar with the definition, but not identical. Also, the SLE mapping of the blade is not unique, so even two machined blades created using different machining software might be different.

A good comparison can be performed by overlapping the geometries of the two blades. The differences will show in variations of color contours that are a function of which blade surface is closer to the camera (Figure 5). Several major observations can be noted. The SLE blade is thicker, but the difference in blade thickness seen at the shroud does not match the one towards the hub. Although there is a slight variation in the design process between the two blades and the SLE has a lower tolerance, being 125 microns thicker, that still does not explain why the two blades match almost perfectly in the hub region and are so different at the shroud. At the pressure side, the curved blade is on top, while things are reversed at the suction side. This attests that the SLE blades have less bowing than the curved blades. Although this is the general trend of the blade, things are more sensitive at the leading edge of the main blade. Here, a totally different 


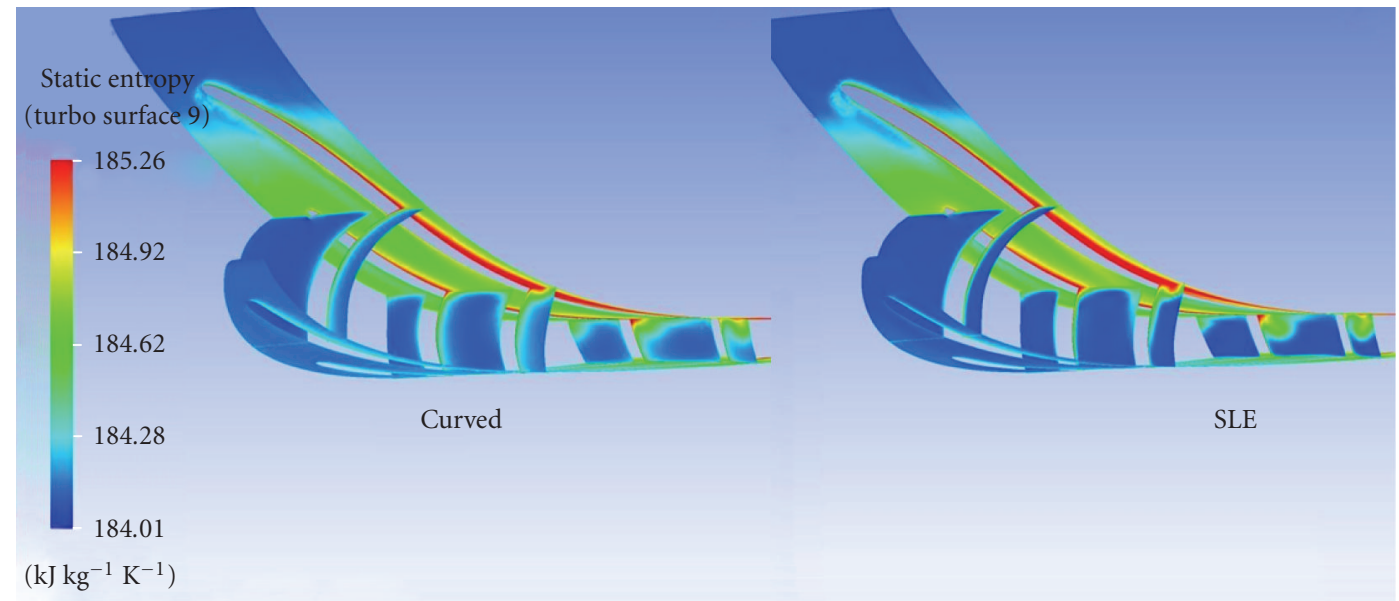

FiguRE 9: Spanwise contour plots of static entropy at three constant streamwise locations at flow factor of 0.0713.

design is seen for the SLE compared to the curved blade. It is expected that this change in design will cause a high variation in the velocity profile from one blade to the other.

A first step towards a deeper investigation of the difference in performance between the two blades is analyzing the velocity blade loading factors at three spanwise positions: hub, midspan, and shroud (Figure 6). As expected from the geometry estimate, the curves are close together along the hub and maintain small deviations from one to the other at midspan. The only significant difference is at the leading edge of the main blade, which is more highly loaded in the SLE case. The cause of this change in loading is the modification of the incidence angle. Progressing towards the shroud, a different variation from the curved blade to the SLE one can be seen. The leading parts of the blades show similar loading profile, but downstream of the splitter changes radically. The SLE splitter is less loaded than the curved one while the opposite is true for the main blade. Different loading profiles can be associated also with a different mass flow rate distribution. The SLE flow passage is distributed as follows: $49.8 \%$ in the main blade pressure side and $50.2 \%$ in the suction side. In the case of the curved blades, the main blade pressure side has a higher mass flow rate $(50.5 \%)$, so the flow division is reversed.

A comparison of the streamwise velocity contours for the shroud and hub (Figure 7) illustrates the impact of blade loading on the flow field. At the shroud leading edge of the main blade, it can be seen that there is a difference in the diffusion of flow along the main blade pressure surface up to the splitter leading edge. The rate of diffusion for the curved blade is greater than that of the SLE blade resulting in a higher blade loading. Downstream of the splitter leading edge, both curved and SLE main blade pressure surfaces show a similar rate of diffusion. On the main blade suction surface, the results are different. Both blades diffuse the flow along the suction surface to a minimum and then the flow begins to accelerate. It is the SLE blade that accelerates the flow to a higher velocity, which minimizes the amount of low velocity fluid across the passage. The velocity distributions along the splitter blade surfaces for both models appear similar, the difference being the rate of diffusion along the blade surfaces.

At the hub, the velocity contours show similar flow distributions for both the curved and SLE blades. There is an almost constant velocity along the pressure surface of the main and splitter blade. Along the suction surface, the flow is accelerating from leading to trailing edge of both the main and splitter blades. The difference between the two models is that the SLE blade begins to accelerate the flow sooner and at a quicker rate along the suction surface. It is this acceleration of the flow along the suction surface at the shroud and hub that reduces the amount of low velocity fluid moving through the blade passages. This reduction in low velocity fluid is an indication of lower loss compared to the curved blade. This can best be illustrated by looking at contours of entropy.

Figure 8 shows the streamwise plots of entropy near the shroud and hub. For both cases along the shroud, a stream of high entropy (in green) can be seen coming off the suction surface of the main blade that corresponds to low velocity fluid. This stream is much smaller for the SLE blade due to the higher rate of accelerating flow along the suction surface toward the blade exit. At the hub, it is evident that the SLE blade's higher rate of acceleration results in a much lower rate of entropy throughout the entire blade passage. What was not evident from the hub velocity contours was how much more efficient the SLE blade is in the area from the main blade leading edge pressure surface to the splitter leading edge.

The impact of these differences in rates of diffusion and acceleration through the blades is illustrated in Figure 9, in which spanwise contour plots of entropy at three constant streamwise locations are shown. It is quite evident that a greater portion of the blade passages for the SLE blade result in much lower entropy, whereas for the curved blade, it can be seen that the layer of high entropy along the suction surface of the blades continues to grow from the inlet to the exit of the blade resulting in a much higher entropy on average through the blade. 


\section{CONCLUSIONS}

Analysis of experimental results originally showed there was a potential difference in impeller geometry. When this difference was confirmed, it was evident that engineers must continue to be involved in the modeling of impeller geometry to insure that the design intent is maintained. This also means understanding the geometry modeling technique of the design code, but at the same time taking into account the impeller fabrication process.

In this case, engineering used an SLE-based design code. Unknowingly, a change in blade profile tolerancing resulted in a difference in thickness between the two models-again leading to the conclusion that engineers must follow the drafting effort.

Engineering would not have guessed that the differences in modeling techniques could have made such a difference in performance, especially since several spanwise layers were provided to drafting. As this study shows, it was the fact that the SLE blade design was closer to the design intent, the goal being to control rates of diffusion through the blade based on design experience to achieve the best efficiency possible.

The authors are not suggesting that SLEs are the highest performing centrifugal impeller definition. On the contrary, various optimization studies have identified arbitrary impeller blade definitions with better performance than their SLE counterpart. But regardless of the blade definition, the $3 \mathrm{D} C A D$ modeling and manufacturing must match the compressor engineers' design intent.

\section{REFERENCES}

[1] H. Pitkänen, H. Esa, P. Sallinen, J. Larjola, H. Heiska, and T. Siikonen, "Time-accurate CFD analysis of a centrifugal compressor," in Proceedings of the 4th International Symposium on Experimental and Computational Aerodynamics of Internal Flows (ISAIF '99), vol. 2, pp. 130-139, Dresden, Germany, AugustSeptember 1999.

[2] M. B. Flathers and G. E. Bache, "Aerodynamically induced radial forces in a centrifugal gas compressor-part 2: computational investigation," in Proceedings of the 41st ASME International Gas Turbine \& Aeroengine Congress, p. 12, Birmingham, UK, June 1996.

[3] A. Koumoutsos, A. Tourlidakis, and R. L. Elder, "Computational studies of unsteady flows in a centrifugal compressor stage," Proceedings of the Institution of Mechanical Engineers, Part A: Journal of Power and Energy, vol. 214, no. 6, pp. 611633, 2000.

[4] S. Shah and J. Bartos, "Confirming centrifugal compressor aerodynamic performance using limited test data combined with computational fluid dynamic techniques," in Proceedings of the 26th Turbomachinery Symposium, pp. 35-41, Houston, Tex, USA, September 1997.

[5] American Society of Mechanical Engineers, "Test Code on Compressors and Exhausters," ASME Power Test Code 10, New York, 1997.

[6] D. M. Tsay, H. C. Chen, and M. J. Her, "A study of five flank machining of centrifugal compressor impellers," Journal of Engineering for Gas Turbines and Power, vol. 124, no. 1, pp. 177181, 2002. 

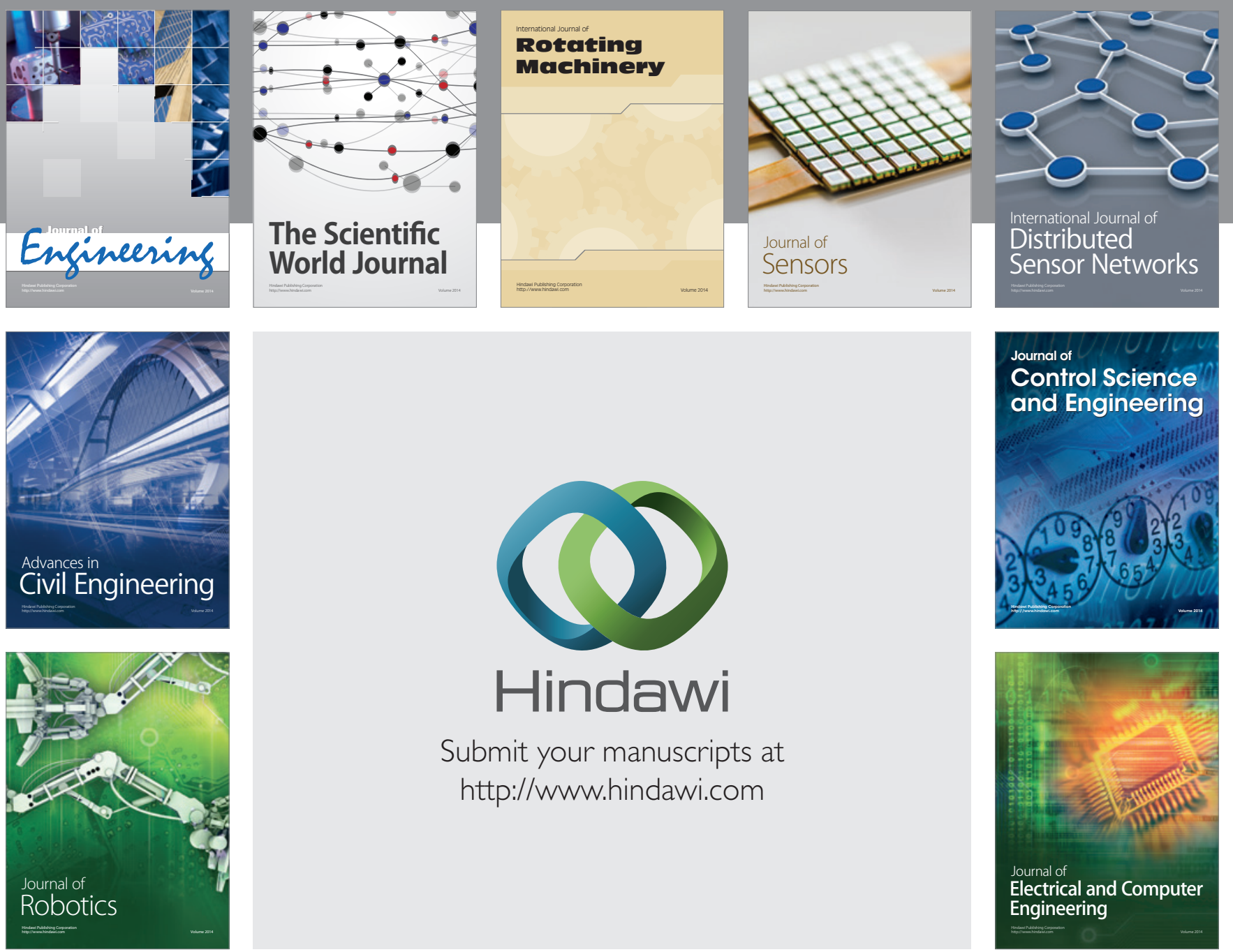

Submit your manuscripts at

http://www.hindawi.com
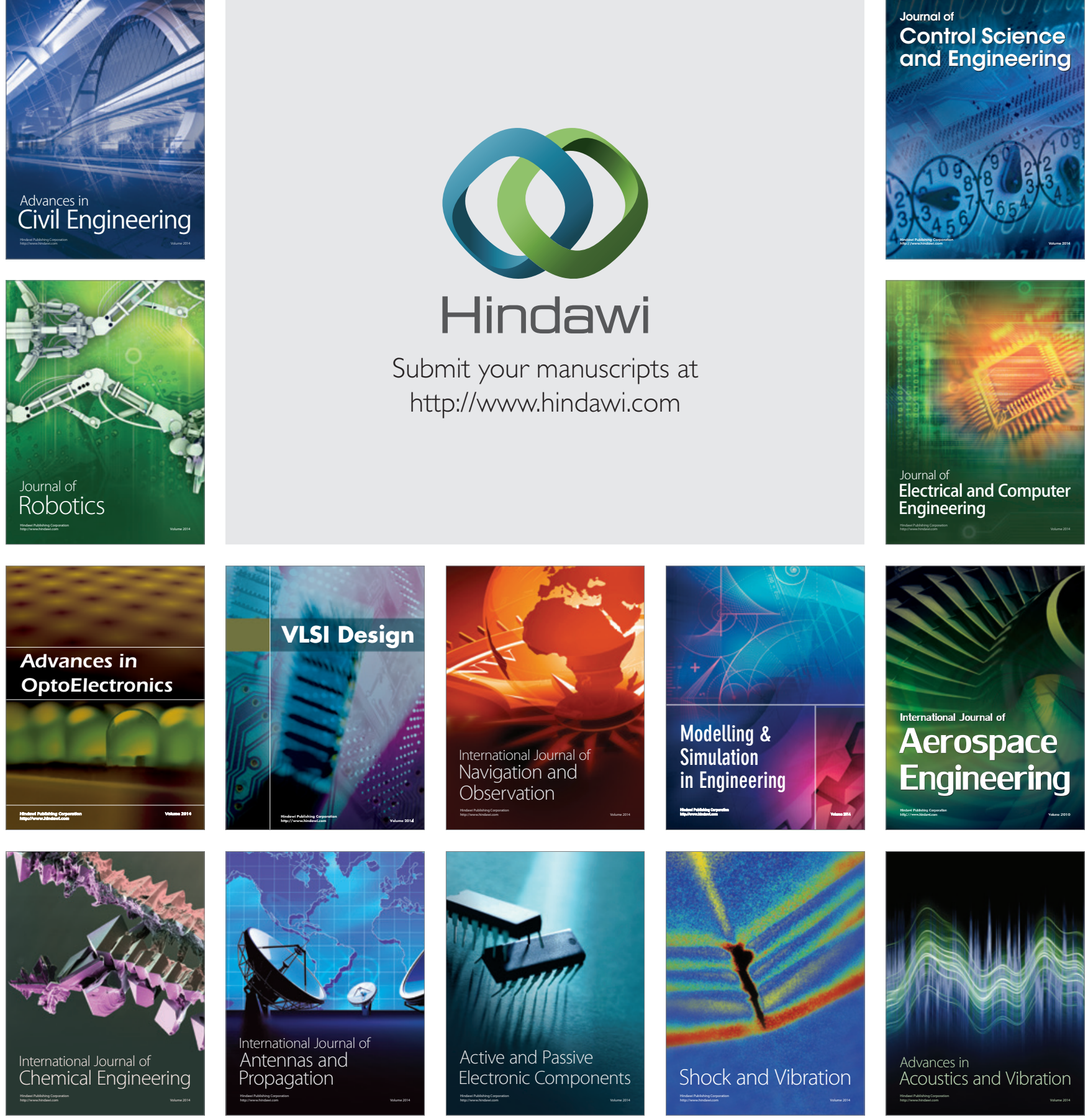